\title{
Erratum to: Integration of land use and land cover inventories for landscape management and planning in Italy
}

\section{Lorenzo Sallustio • Michele Munafò • Nicola Riitano • \\ Bruno Lasserre • Lorenzo Fattorini • Marco Marchetti}

Published online: 29 February 2016

(C) Springer International Publishing Switzerland 2016

\section{Erratum to: Environ Monit Assess \\ DOI 10.1007/s10661-015-5056-7}

The accepted version of Tables 7 and 8 for this published online article contained an error. The corrected Tables 7 and 8 are shown in the next page.

The online version of the original article can be found under doi:10.1007/s10661-015-5056-7.

L. Sallustio $(\bowtie) \cdot$ B. Lasserre $\cdot$ M. Marchetti

Dipartimento di Bioscienze e Territorio, Università degli Studi del Molise, Contrada Fonte Lappone snc, 86090 Pesche, IS, Italy e-mail: lorenzo.sallustio@unimol.it

B. Lasserre

e-mail: lasserre@unimol.it

M. Marchetti

e-mail: marchettimarco@unimol.it

M. Munafò

Italian National Institute for Environmental Protection and Research (ISPRA), Via V. Brancati 48, I-00144 Rome, Italy e-mail: michele.munafo@isprambiente.it
M. Munafò

Department of Civil, Building and Environmental Engineering, Sapienza University of Rome, Via Eudossiana 18, I-00184 Rome, Italy

N. Riitano

Dipartimento di Architettura e Progetto, Facoltà di Architettura, Università di Roma "Sapienza", Via Flaminia 359, 00196 Rome, Italy

e-mail: nicola.riitano@gmail.com

L. Fattorini

Department of Economic and Statistics, University of Siena, Piazza San Francesco 8, 53100 Siena, Italy

e-mail: lorenzo.fattorini@unisi.it 
Table 7 Matrix of size estimates of LC changes that occurred from 2000 to 2012 in the Molise region (values in hectares)

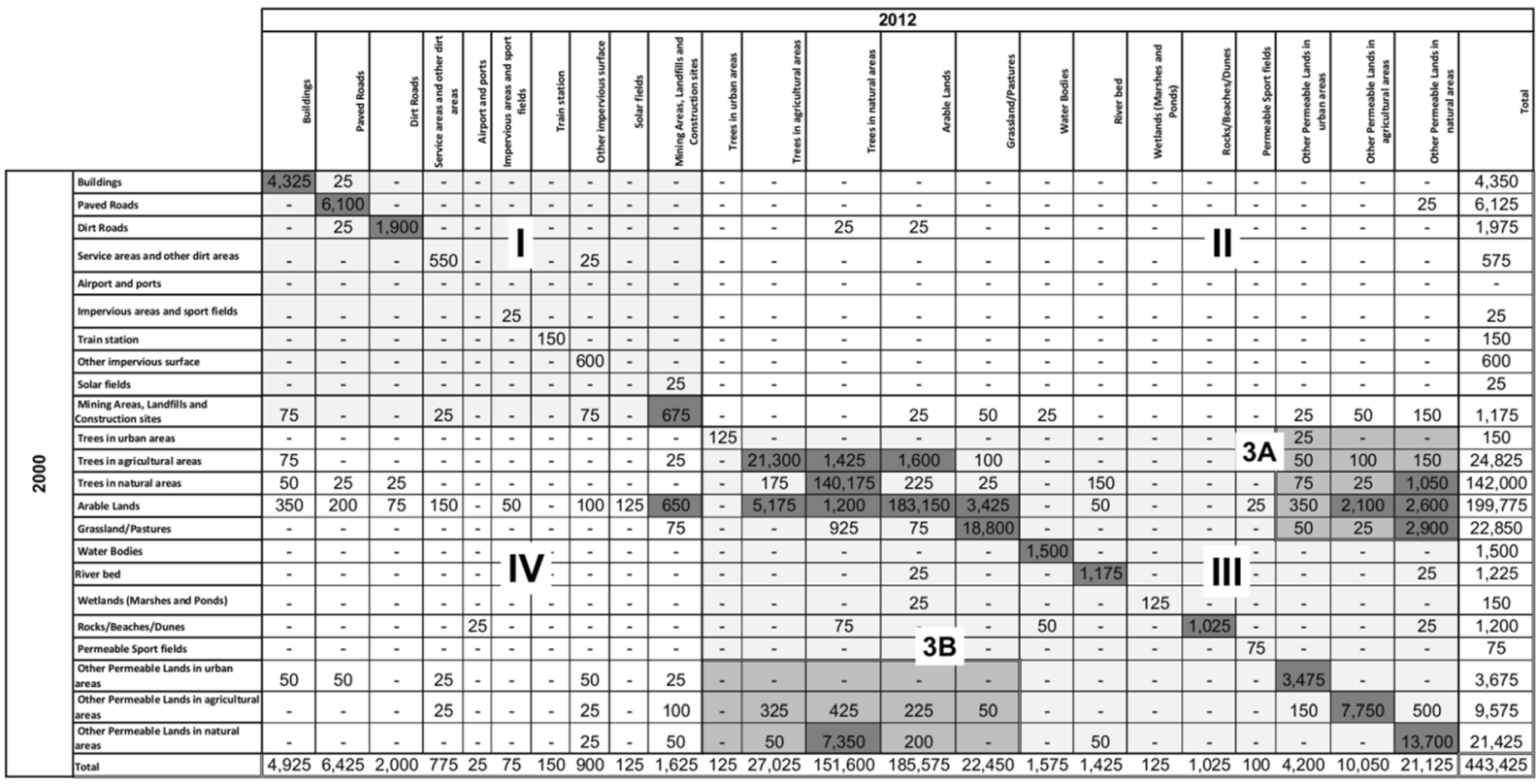

The highlighted cells in grey refer to change estimates greater than 625 ha, with an estimated standard error smaller than $20 \%$

Table 8 Matrix of size estimates of LC changes that occurred from 2000 to 2012 in the Molise region (values as percentages of the network surface)

\begin{tabular}{|c|c|c|c|c|c|c|c|c|c|c|c|c|c|c|c|c|c|c|c|c|c|c|c|c|c|}
\hline & \multicolumn{24}{|c|}{2012} \\
\hline & & 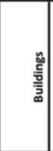 & 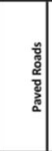 & 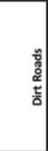 & 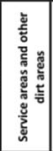 & 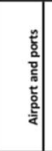 & 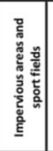 & $\begin{array}{l}\frac{\mathbf{i}}{2} \\
\frac{\mathrm{n}}{2}\end{array}$ & 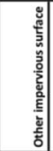 & $\begin{array}{l}\frac{n}{0} \\
\frac{a}{0} \\
\frac{a}{0}\end{array}$ & 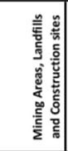 & 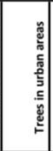 & | & 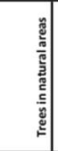 & $\begin{array}{l}\frac{i}{3} \\
\frac{a}{2}\end{array}$ & 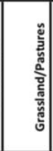 & 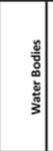 & $\begin{array}{l}\text { : } \\
\vdots \\
\frac{0}{2}\end{array}$ & 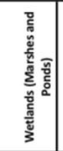 & 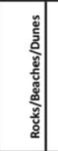 & 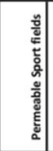 & 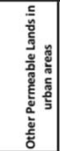 & 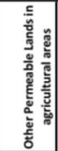 & 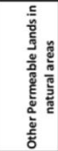 & 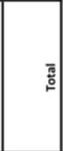 \\
\hline \multirow{24}{*}{ : } & Builidings & 0.94 & 0.01 & - & - & - & - & - & - & - & - & - & - & - & - & - & - & - & - & - & - & - & - & - & 0.95 \\
\hline & \begin{tabular}{|l|} 
Paved Roads \\
\end{tabular} & - & 1.33 & - & - & - & - & - & - & - & - & $\cdot$ & $\cdot$ & - & $\cdot$ & $\cdot$ & - & - & - & - & - & - & - & 0.01 & 1.34 \\
\hline & \begin{tabular}{|l|} 
Dirt Roads \\
\end{tabular} & - & 0.01 & 0.41 & - & - & -1 & - & - & - & - & - & - & 0.01 & 0.01 & - & - & - & - & & $=$ & - & - & - & 0.43 \\
\hline & Service areas and other ditr areas & - & - & - & 0.12 & - & $\therefore$ & - & 0.01 & - & - & - & . & - & - & - & - & - & - & . & - & - & - & . & 0.13 \\
\hline & \begin{tabular}{|l|} 
Airport and ports \\
\end{tabular} & - & - & - & - & - & - & - & - & - & - & - & - & - & - & - & - & - & - & - & - & - & - & - & - \\
\hline & Impenious sreas and sport fieds & - & - & - & - & - & 0.01 & - & - & - & - & - & - & - & - & - & - & - & - & - & - & - & - & - & 0.01 \\
\hline & \begin{tabular}{|l|} 
Train station \\
\end{tabular} & - & - & - & - & - & - & 0.03 & - & - & - & - & - & - & - & - & - & - & - & - & - & - & - & - & 0.03 \\
\hline & \begin{tabular}{|l|} 
Other impenious surface \\
\end{tabular} & - & - & - & - & - & - & - & 0.13 & - & - & - & - & - & - & - & - & - & - & - & - & - & - & - & 0.13 \\
\hline & \begin{tabular}{|l|} 
Solar fields \\
\end{tabular} & - & - & - & - & - & - & - & - & - & 0.01 & - & - & - & - & - & - & - & - & - & - & - & - & - & 0.01 \\
\hline & 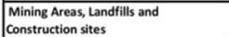 & 0.02 & - & - & 0.01 & - & - & - & 0.02 & - & 0.15 & - & - & - & 0.01 & 0.01 & 0.01 & - & - & - & - & 0.01 & 0.01 & 0.03 & 0.26 \\
\hline & \begin{tabular}{|l} 
Trees in urban areas \\
\end{tabular} & - & - & - & - & - & - & - & - & - & - & 0.03 & - & - & - & - & - & - & - & - & & 0.01 & - & - & 0.03 \\
\hline & \begin{tabular}{|l|l} 
Trees in agricultural areas \\
\end{tabular} & 0.02 & - & - & - & - & - & - & - & - & 0.01 & - & 4.65 & 0.31 & \begin{tabular}{|l|}
0.35 \\
\end{tabular} & 0.02 & - & - & - & - & $3 \mathrm{~A}$ & 0.01 & 0.02 & 0.03 & \begin{tabular}{|l|}
5.41 \\
\end{tabular} \\
\hline & \begin{tabular}{|l|l} 
Trees in natural areass \\
\end{tabular} & 0.01 & 0.01 & 0.01 & - & - & - & - & - & - & - & - & 0.04 & 30.57 & 0.05 & 0.01 & - & 0.03 & - & - & - & 0.02 & 0.01 & 0.23 & 30.97 \\
\hline & \begin{tabular}{|l|} 
Arable Lands \\
\end{tabular} & 0.08 & 0.04 & 0.02 & 0.03 & - & 0.01 & - & 0.02 & 0.03 & 0.14 & - & 1.13 & 0.26 & 39.94 & 0.75 & - & 0.01 & - & - & 0.01 & 0.08 & 0.46 & 0.57 & 43.57 \\
\hline & \begin{tabular}{|l|} 
Grassland/Pastures \\
\end{tabular} & - & - & - & - & - & - & - & - & - & 0.02 & - & - & 0.20 & 0.02 & 4.10 & - & - & - & - & - & 0.01 & 0.01 & 0.63 & 4.98 \\
\hline & \begin{tabular}{|l|l|} 
Water Bodies \\
\end{tabular} & $\cdot$ & - & $\cdot$ & $\cdot$ & - & & - & $\cdot$ & $\cdot$ & $\cdot$ & $\cdot$ & $\cdot$ & - & $\cdot$ & $\cdot$ & 0.33 & $\cdot$ & $\cdot$ & & & - & - & - & 0.33 \\
\hline & River bed & - & - & - & - & - & I & 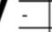 & - & - & - & - & - & - & 0.01 & - & - & 0.26 & - & & II & - & - & 0.01 & 0.27 \\
\hline & Wettands (Marshes and Ponds) & - & - & - & - & - & - & - & - & - & - & - & - & - & 0.01 & - & - & - & 0.03 & - & - & - & - & - & 0.03 \\
\hline & \begin{tabular}{|l|} 
Rocks/Beaches/Dunes \\
\end{tabular} & - & - & $\cdot$ & - & \begin{tabular}{l|l|l}
0.01 & \\
\end{tabular} & - & - & - & - & - & - & - & 0.02 & \multirow{2}{*}{\multicolumn{2}{|c|}{$3 B$}} & \begin{tabular}{|l|}
0.01 \\
\end{tabular} & - & - & 0.22 & - & - & - & 0.01 & 0.26 \\
\hline & \begin{tabular}{|l} 
Permeable Sport fields \\
\end{tabular} & - & - & - & - & - & - & - & - & - & - & - & - & - & & & - & - & - & - & 0.02 & - & - & $\cdot$ & 0.02 \\
\hline & Other Permeable Lands in urban areas & 0.01 & 0.01 & - & 0.01 & - & . & . & 0.01 & - & 0.01 & . & - & - & - & - & - & . & - & - & - & 0.76 & - & . & 0.80 \\
\hline & \begin{tabular}{|l}
$\begin{array}{l}\text { Oaher Permeable Lands in agricultural } \\
\text { areas }\end{array}$ \\
\end{tabular} & - & - & - & 0.01 & - & - & - & 0.01 & - & 0.02 & - & 0.07 & 0.09 & 0.05 & 0.01 & - & - & - & - & - & 0.03 & 1.69 & 0.11 & 2.09 \\
\hline & Other Permeable Lands in natural areas & . & . & . & . & . & . & - & 0.01 & . & 0.01 & . & 0.01 & 1.60 & 0.04 & - & . & 0.01 & . & . & . & . & . & 2.99 & 4.67 \\
\hline & Total & 1.07 & 1.40 & 0.44 & 0.17 & 0.01 & 0.02 & 0.03 & 0.20 & 0.03 & 0.35 & 0.03 & 5.89 & 33.06 & 40.47 & 4.90 & 0.34 & 0.31 & 0.03 & 0.22 & 0.02 & 0.92 & 2.19 & 4.61 & 96.71 \\
\hline
\end{tabular}

The cells highlighted in grey refer to change estimates greater than $0.14 \%$, with an estimated standard error smaller than $20 \%$ 